\title{
Consultoria e ligação em saúde mental na perspectiva da equipe da Estratégia de Saúde da Família*
}

\author{
Ana Carolina Guidorizzi Zanetti ${ }^{1}$ \\ (iD) https://orcid.org/0000-0003-0011-4510 \\ Sueli Aparecida Frari Galera ${ }^{1}$ \\ (D) https://orcid.org/0000-0001-7974-9214 \\ Jacqueline de Souza ${ }^{1}$ \\ (D) https://orcid.org/0000-0002-6094-6012 \\ Kelly Graziani Giacchero Vedana ${ }^{1}$ \\ (iD) https://orcid.org/0000-0001-7363-2429 \\ Edilaine Cristina da Silva Gherardi-Donato ${ }^{1}$ \\ (D) https://orcid.org/0000-0001-7475-6650 \\ Margarita Antonia Villar Luis ${ }^{1}$ \\ (iD) https://orcid.org/0000-0002-9907-5146 \\ Lisa Laredo de Camargo ${ }^{1,2}$ \\ (iD) https://orcid.org/0000-0001-9452-5761 \\ João Mazzoncini de Azevedo Marques ${ }^{3}$ \\ (iD) https://orcid.org/0000-0002-3100-3883
}

\footnotetext{
* Apoio financeiro do Conselho Nacional de Desenvolvimento Científico e Tecnológico (CNPq), Brasil, processo no 575255/2008-0.

${ }^{1}$ Universidade de São Paulo, Escola de Enfermagem de Ribeirão Preto, Centro Colaborador da OPAS/OMS para o Desenvolvimento da Pesquisa em Enfermagem, Ribeirão Preto, SP, Brasil.

${ }^{2}$ Bolsista da Coordenação de Aperfeiçoamento de Pessoal de Nível Superior (CAPES), Brasil.

3 Universidade de São Paulo, Faculdade de Medicina de Ribeirão Preto, Ribeirão Preto, SP, Brasil.
}

Objetivo: analisar as atividades de consultoria e ligação, realizadas pelos profissionais especializadosem Saúde Mental, na perspectiva dos profissionais da ESF. Método: estudo exploratório, realizado em cinco núcleos de Saúde da Família, em um município do interior paulista, em 2010. Os dados foram obtidos por meio de entrevistas, os quais foram submetidos à Análise de Conteúdo. Resultados: as atividades de consultoria e ligação mostraram-se como um espaço de aprendizado mútuo entre especialistas de Psiquiatria e Saúde Mental e equipes da ESF. Conclusão: recomenda-se a ampliação do número de atividades que articulem as ações de saúde mental com a ESF, como a consultoria e ligação, a fim favorecer a sensibilidade das equipes para as demandas de saúde mental na ESF e consolidação da integralidade do cuidado.

Descritores: Saúde Mental; Atenção Primária à Saúde; Estratégia de Saúde da Família; Saúde da Família.

\section{Como citar este artigo}

Zanetti ACG, Galera SAF, Souza J, Vedana KGG, Gherardi-Donato ECS, Luis MAV, et. al. Mental health consultation-liaison from the perspective of the primary health care team. SMAD, Rev Eletrônica Saúde Mental Álcool Drog. 2019;15(3):1-8. doi: https://dx.doi.org/10.11606/issn.1806-6976.smad.2019.151548 


\section{Mental health consultation-liaison from the perspective of the primary health care team}

Objective: analyze the consulting and liaison activities carried out by professionals specialized in mental health from the perspective of the PHC. Method: an exploratory study, realized in five Family Health centers, in a municipality in the countryside of São Paulo, in 2010. Data were collected through interviews, which were submitted to content analysis. Results: as consultationliaison activities were shown as a space for mutual learning between specialists in psychiatry and mental health and PHC teams. Conclusion: it is recommended to increase the number of activities that articulate mental health actions with PHC, such as consultation and liaison, in order to favor the sensitivity of the teams to the demands of mental health in the PHC and consolidation of the integral care.

Descriptors: Mental Health; Primary Health Care; Family Health Strategy; Family Health.

\section{Consultoría y conexión en salud mental en la perspectiva de la estrategia de salud de la familia}

Objetivo: analisar como actividades de consultoría y ligação realizadas pelis profissionais tourism em saúde mental na perspective dos profissionais da ESF. Método: estudo exploratorio, realizado en cinco núcleos de Salud de la familia, en el municipio de interior paulista, en 2010. Os dados foram obtidos por meio de entrevistas, os quais foram submetidos à análise de conteúdo. Resultados: como atividades de consultoría y ligação mostraram-se como um espaço de aprendised mútuo entre especialistas de psiquiatria e saúde mental e equipes del ESF. Conclusión: recomendar una ampliación del número de actividades que articulan como funciones de salud mental con un ESF, como una consultoría y ligazón, un enfoque para sensibilizar a los equipos para las demandas de salud mental en ESF y consolidación de la integridad del cuidado.

Descriptores: Salud Mental; Atención Primaria de Salud; Estrategia de Salud Familiar; Salud de la Familia. 


\section{Introdução}

As condições para que o Sistema Único de Saúde seja consolidado como um modelo de saúde passa pela implementação efetiva da rede de Atenção Primária à Saúde (APS), particularmente por meio da Estratégia da Saúde da Família (ESF). Dentro do processo de trabalho da ESF, o conceito de territorialização, campo que delimita a ação da Unidade de Saúde quanto ao espaço e população, é fundamental(1). Os profissionais da equipe da ESF devem realizar a busca ativa das pessoas inseridas em um território delimitado, identificando aqueles com problemas de saúde, incluindo problemas psicossociais e transtornos mentais propriamente ${ }^{(1)}$. Reconhece-se que, na APS, a prevalência de problemas psicossociais é da ordem de $40 \%$ e de transtornos mentais, $25 \%{ }^{(2)}$. Estudo realizado no Rio Grande do Sul mostrou que a prevalência de transtornos mentais de indivíduos atendidos em um serviço de APS foi de aproximadamente $51 \%{ }^{(3)}$. Para a maioria desses indivíduos, o único cuidado ofertado é aquele realizado pelos profissionais das equipes de $\operatorname{ESF}^{(3)}$.

Estudos mostram que o conjunto dos serviços/ profissionais especializados em saúde mental, mesmo em países de alta renda com sistemas universais de saúde, atendem, no máximo, a $3 \%$ da população anualmente, enquanto que a prevalência anual dos transtornos mentais gira ao redor dos 30\%, o que aponta importantes lacunas entre a demanda e a oferta de atendimento nessa área(4-5). A Organização Mundial da Saúde (OMS) reconhece que os serviços de APS podem funcionar como importantes articuladores das redes de saúde mental (SM), já que, devido à sua proximidade com as comunidades, as famílias e os indivíduos, têm o potencial de detectar mais rapidamente problemas de saúde mental e fornecer intervenções adequadas às características singulares e contextuais de cada situação (inclusive, por meio de encaminhamento para serviços especializados, quando necessário)(6-7).

No entanto, estudos mostram que as equipes da APS fazem a detecção de aproximadamente $50 \%$ das pessoas com problemas de saúde mental dentro no seu território e que, mesmo quando o problema é detectado, a taxa de tratamento adequado é baixa(6). No Brasil, apesar dos esforços empreendidos nos últimos anos, os profissionais das equipes das ESF frequentemente ainda carecem de capacitação para o desenvolvimento de ações em saúde mental. Além disso, há dificuldades na capacidade de desenvolver ações que se estendam ao espaço social mais amplo(8).

Desde 1999, em uma cidade do interior paulista, profissionais de saúde, vinculados aos cursos de Medicina e Enfermagem de uma universidade pública, iniciaram ações de cuidado integrado de saúde mental nos serviços de ESF, cuidado esse caracterizado por encontros periódicos e facilitação da comunicação entre os profissionais desses serviços e profissionais especializados em saúde mental, com o objetivo de fornecer apoio às equipes da ESF no manejo de situações envolvendo problemas psicossociais e/ou transtornos mentais e, consequentemente, na sua capacitação em saúde mental.

Os referenciais teórico-práticos para essas ações de cuidado integrado em saúde mental foram experiências britânicas, canadenses e norte-americanas de consultoria e ligação em saúde mental na $\operatorname{APS}^{(9-12)}$. Esse conceito de consultoria e ligação em saúde mental em APS é caracterizado por: encontros periódicos entre profissionais das equipes de APS e profissionais especializados, os quais podem incluir atendimentos conjuntos de pacientes e discussões de casos e de temas solicitados por essas equipes; comunicação facilitada entre os profissionais também por meio de contatos via telefone e/ou internet; ajuda dos profissionais especializados em saúde mental no encaminhamento de pacientes para atendimento especializado fora da APS e no fornecimento de informações sobre esse atendimento para a equipe de cuidados primários. Assim sendo, esse modelo de consultoria e ligação na APS mantém o papel central dos profissionais/equipes de APS como provedores de cuidado para a grande maioria das pessoas acompanhadas nesse nível. Os profissionais especializados em saúde mental descritos na literatura como atuantes desse modelo são diversos: enfermeiros, psicólogos, médicos ou assistentes sociais ${ }^{(6,9,11,13-15)}$.

Por exemplo, a função de profissionais de Enfermagem na qualidade de consultores em saúde mental foi instituída desde a década de 70, em países desenvolvidos, e ainda se encontra em processo de consolidação em países em desenvolvimento(16). Acredita-se que o envolvimento do enfermeiro em atividades de consultoria pode facilitar a resolução de questões de menor complexidade que envolvem a saúde mental, agilizar a identificação dos casos de transtornos mentais com maior acurácia, favorecer a oferta de cuidados de menor complexidade, favorecer a qualidade de vida da família do usuário do serviço de saúde e melhorar a articulação com os outros serviços de saúde. Também, pode promover a capacitação e a supervisão da equipe de Enfermagem e o reconhecimento dos vínculos familiares e sociais estabelecidos entre os profissionais de saúde e a comunidade ${ }^{(16-17)}$.

No Brasil, a partir de 2003, inicia-se o delineamento das primeiras diretrizes para a aproximação da saúde mental com a ESF, com a publicação do documento Apoio Matricial da Saúde Mental às equipes da ESF. O Apoio Matricial da Saúde institui o modelo de consultoria e ligação pautado no vínculo terapêutico. Esse modelo valoriza as ações terapêuticas no âmbito do território, 
enfatiza a singularidade do indivíduo, evitando a fragmentação do cuidado, e considera as dimensões familiar, sociocultural, econômica e biopsicossocial que envolvem o cotidiano das ações para a promoção de saúde mental(18).

Nas literaturas nacional e internacional, há reconhecimento de que, embora os órgãos que gerem a saúde no Brasil e no mundo destaquem que as equipes da ESF têm papel chave na prevenção e manutenção de problemas de saúde mental, existem lacunas de conhecimento sobre como as equipes ESF se articulam para atender às demandas de saúde mental(7,19-20).

Embora haja uma perspectiva otimista em relação às atividades de consultoria e ligação entre um serviço especializado de saúde mental para as equipes da ESF frente às possibilidades de acesso facilitado, diagnóstico precoce de problemas, início das intervenções apropriadas e imediatas centradas na pessoa e criação de um ambiente não estigmatizante, os profissionais da ESF nem sempre se sentem ou estão preparados para atender às necessidades em saúde mental.

As justificativas deste estudo foram as dificuldades frequentes descritas por equipes/profissionais de APS para manejar problemas de saúde mental e a experiência adquirida no trabalho de consultoria e ligação desenvolvido em uma cidade do interior paulista para lidar com essa questão.

\section{Objetivos}

Este estudo teve como objetivo analisar as atividades de consultoria e ligação, realizadas pelos profissionais especializados em Saúde Mental e Psiquiatria, na perspectiva dos profissionais da ESF.

\section{Método}

Trata-se de um estudo descritivo e exploratório realizado junto a cinco equipes de ESF do município de Ribeirão Preto, ligadas à Universidade de São Paulo, em 2010. Os profissionais dessas equipes tinham acesso às atividades de consultoria e ligação das quais participaram um médico e uma enfermeira, especializados em Psiquiatria e Saúde Mental. As atividades de consultoria e ligação abrangiam: discussões de temas e de casos; atendimento conjunto, incluindo visitas domiciliares; atendimento dos profissionais especializados sem participação simultânea de profissionais da APS; discussão de aspectos organizacionais e do processo de trabalho da equipe de ESF; encaminhamento rápido para o serviço ambulatorial especializado de nível secundário, onde também trabalhavam o psiquiatra e a enfermeira de saúde mental, se assim decidido consensualmente com a equipe de APS; facilitação do encaminhamento para outros serviços especializados, como o Hospital-Dia, Centro de Atenção Psicossocial para Usuários de Álcool e Drogas e internação integral psiquiátrica.

O projeto foi aprovado pelo Comitê de Ética e Pesquisa, em 15 de julho de 2009, sob o protocolo no 17/2009, de acordo com a resolução CNS 466/96(21). Os profissionais de saúde foram recrutados nos núcleos de Saúde da Família após a aprovação do projeto pelo comitê de ética em pesquisa. Foram convidados a participar do estudo 58 profissionais dessas cinco equipes da ESF. Sete se recusaram a participar e um se desligou do serviço antes de ser agendada sua entrevista. Então, uma amostra por conveniência final foi constituída por 50 profissionais de saúde.

Para a coleta de dados, utilizou-se um roteiro estruturado contendo as variáveis sociodemográficas (sexo, idade, instituição onde realizou a formação, formação na área de Saúde Mental e Psiquiatria, local e tempo de trabalho atual) e um roteiro de entrevista semiestruturado contendo a seguinte questão norteadora: "Como você se sente quanto ao trabalho de consultoria e ligação em Saúde Mental e Psiquiatria no seu serviço?".

Os dados foram obtidos por meio de entrevistas, em horário pré-determinado pelos participantes, em salas reservadas nos referidos núcleos. Primeiramente, esclareceram-se os objetivos e a natureza do estudo e, após a concordância e a assinatura do Termo de Consentimento Livre e Esclarecido, deu-se início à entrevista. O tempo médio para a realização das entrevistas foi de trinta minutos e, ao término, o entrevistador se colocava à disposição para esclarecimentos adicionais. O período de coleta de dados foi de julho de 2009 a janeiro de 2010.

As entrevistas foram gravadas, transcritas e os dados que poderiam identificar o profissional foram retirados. Após esta etapa, os dados foram submetidos à Análise de Conteúdo, segundo Bardin(22). As entrevistas foram lidas visando a identificar similaridades e diferenças nos conteúdos expressos pelos participantes com o intuito de identificar as atividades de consultoria e ligação, realizadas pelos profissionais especializados em Saúde Mental e Psiquiatria, na perspectiva das equipes da Estratégia da Saúde da Família. A codificação das unidades de significado foi realizada por dois pesquisadores tendo como norte as ações de consultoria e ligação descritas em estudos anteriores ${ }^{(9-11)}$. Após essa etapa, os códigos foram agrupados nas seguintes categorias: Demandas de saúde mental na ESF e o papel da consultoria e Ligação como elemento de apoio na ESF. Os parâmetros para a análise dos resultados foram as recomendações para a operacionalização do apoio matricial na Atenção Primária à Saúde ${ }^{(23-24)}$. 


\section{Resultados}

Dos 50 (100\%) profissionais das equipes da ESF, 19 (38\%) eram agentes comunitários de saúde; mulheres, 39
(78\%); com média de idade de 34 anos ( $\mathrm{DP}=8,6)$ e tempo de trabalho médio de 48 meses ( $D P=35,5)$. A totalidade dos investigados não tinha formação anterior como especialista em Saúde Mental, como é possível analisar na Tabela 1.

Tabela 1 - Caracterização dos profissionais que participaram do estudo $(n=50)$. Ribeirão Preto - SP, Brasil, 2010

\begin{tabular}{|c|c|c|c|c|}
\hline Variáveis & $\begin{array}{c}\text { Profissionais } \\
\mathrm{n}(\%)\end{array}$ & $\begin{array}{l}\text { Média } \\
\text { (D.P.) }\end{array}$ & Mediana & Intervalo \\
\hline \multicolumn{5}{|l|}{ Ocupação } \\
\hline Agente Comunitário de Saúde & $19(38)$ & & & \\
\hline Auxiliar de Enfermagem & $5(10)$ & & & \\
\hline Enfermeiro & $6(12)$ & & & \\
\hline Médico residente & $15(30)$ & & & \\
\hline Médico contratado & $5(10)$ & & & \\
\hline \multicolumn{5}{|l|}{ Sexo } \\
\hline Feminino & $39(78)$ & & & \\
\hline Masculino & $11(22)$ & & & \\
\hline Idade (anos) & & $34,4(8,6)$ & 31 & $23-58$ \\
\hline Tempo de trabalho (meses) & & $47,6(35,5)$ & 35,5 & $5-12$ \\
\hline \multicolumn{5}{|l|}{ Formação em SM* } \\
\hline Sim & - & & & \\
\hline Não & $50(100)$ & & & \\
\hline
\end{tabular}

A seguir, apresentam-se as duas categorias de análise extraídas dos depoimentos dos participantes: Demandas de saúde mental na ESF e o papel da consultoria e Ligação como elemento de apoio na ESF.

A primeira categoria de análise, denominada Demandas de saúde mental na ESF e o papel da consultoria, mostrou que profissionais da ESF se deparam com várias demandas de saúde mental dos usuários dos serviços de saúde. Essas demandas estão relacionadas às necessidades dos usuários frente ao elevado número de casos e problemas inerentes aos diversos diagnósticos de transtornos mentais (depressão e ansiedade relacionadas ao uso de substâncias, esquizofrenia, transtorno bipolar, demência, etc.), conforme se segue.

...muitos casos de depressão e ansiedade. (RESIDENTE); ...os problemas ou situações são várias. [...] os mais comuns são depressão e transtorno de ansiedade. (MÉDICO); problemas como depressão, ansiedade, também, e um monte de coisa. (AGENTE COMUNITÁRIO DE SAÚDE); ...cerca de $70 \%$ da população tem alguma demanda relacionada à saúde mental. (ENFERMEIRO)

Os depoimentos também mostram que há situações de vulnerabilidade social, tais como baixa autoestima, pouco acesso à informação, dificuldades de interação entre membros da família, violência doméstica e conjugal, abuso e negligência infantil e tentativas e/ou concretização do suicídio, que demandam suporte de profissional de saúde capacitado e com especialização em Psiquiatria e Saúde Mental. agressão física e verbal de mãe com filho, conflitos familiares. (AGENTE COMUNITÁRIO DE SAÚDE); ...então, os problemas são vários, pessoas que têm problema com a autoestima, pessoas que têm pouca informação. (AGENTE COMUNITÁRIO DE SAÚDE)

Nos depoimentos, constata-se que as atividades de consultoria e ligação se dão por meio de contato direto dos especialistas em Saúde Mental nos atendimentos em conjunto com a equipe do serviço. As falas mostram que os profissionais de saúde reconhecem a consultoria e ligação como elemento de apoio na ESF. Esse reconhecimento foi constatado em todas as categorias profissionais investigadas, independentemente do nível de formação.

Desse modo, entende-se que as atividades de consultoria e ligação, na perspectiva dos profissionais da APS, aumentaram sua segurança no manejo das demandas de saúde mental, contribuíram para a sua educação continuada, ampliaram o seu repertório de conhecimento teórico e prático e favoreceram a sua a capacidade resolutiva.

....no decorrer dos anos, a gente vem sendo preparado pela consultoria e isto é muito importante. (ENFERMEIRO); ...no começo, eu tinha muita insegurança, (...) mas, com as consultorias aqui, tanto no estágio, quanto na Unidade de Emergência, hoje eu tenho mais segurança, muito mais do que quando comecei. (RESIDENTE); ...com a consultoria e ligação, nós aprendemos a entrevistar, manejar, ajustar os medicamentos, acho que ajuda muito mesmo, faz muita diferença.(MÉDICO); A gente passa a ter um olhar diferente para a saúde mental na ESF (ENFERMEIRO), podendo ser verificado na Figura 1, abaixo 


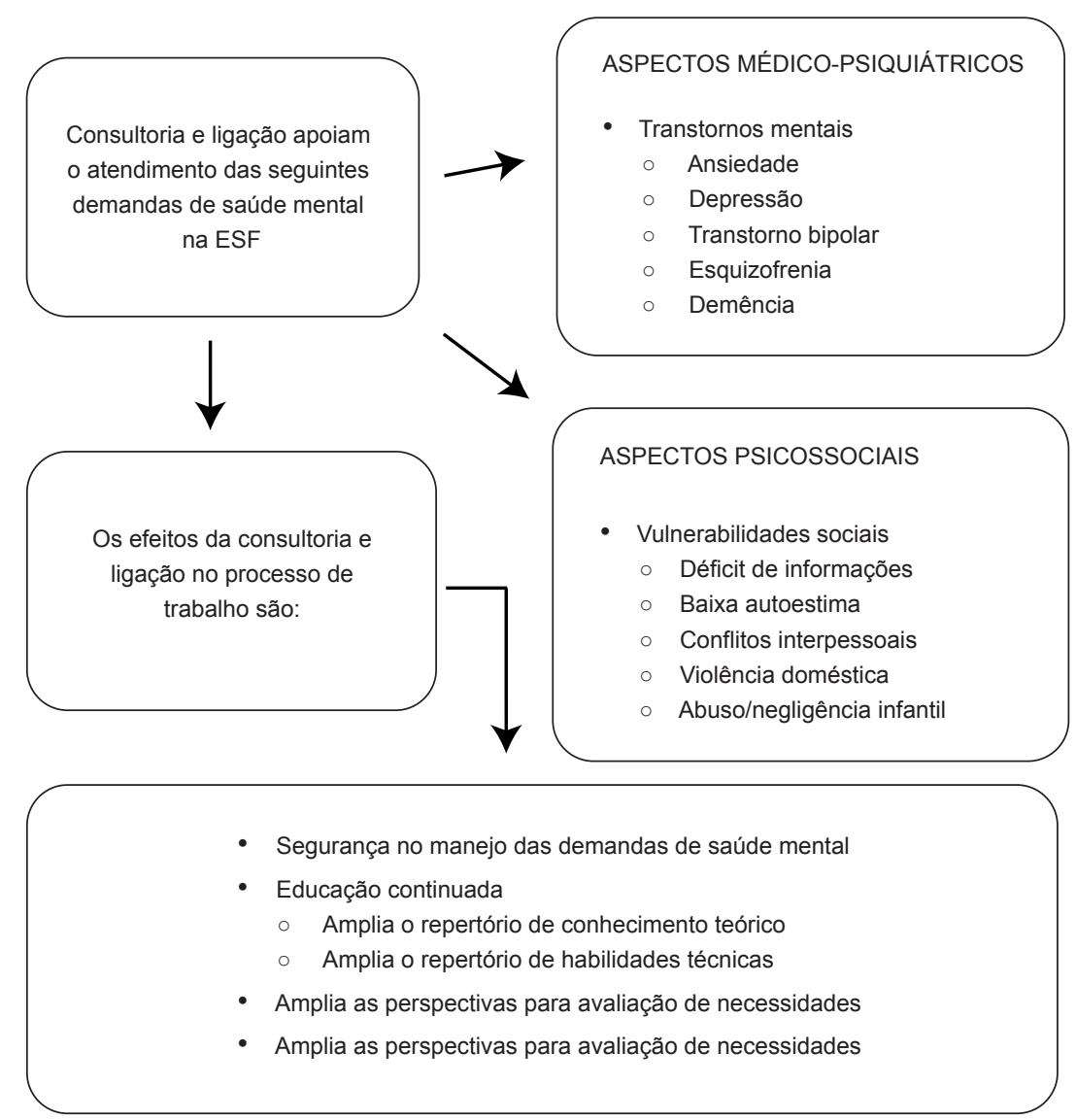

Figura 1 - Síntese das atividades de consultoria e ligação, realizadas pelos profissionais especializados em Saúde Mental e Psiquiatria, na perspectiva dos profissionais das equipes ESF

\section{Discussão}

Ao analisar as atividades de consultoria e ligação, realizadas pelos profissionais especializados em Saúde Mental, na perspectiva dos profissionais da ESF, constata-se que os participantes reconhecem as atividades de consultoria e ligação como essenciais para auxiliar no manejo tanto de demandas específicas de saúde mental (como transtornos mentais e suas manifestações), quanto de situações de vulnerabilidade social. As atividades de consultoria e ligação estimulavam as ações terapêuticas que valorizavam as singularidades das pessoas, ajudando a pensar sobre ações que lidassem não só com a dimensão biológica e com diagnóstico médico-psiquiátrico, mas, também, com aspectos sociais (família, vizinhança, aspectos econômicos, etc.) e psicológicos (a subjetividade, a experiência vivida pela pessoa). Os resultados corroboram outros estudos que enfatizam o caráter biopsicossocial, holístico e centrado nas pessoas que o apoio matricial tem potência para assumir(20,26). Por exemplo, estudo que avaliou o apoio matricial em saúde mental no município de Porto Alegre, em duas equipes da ESF, mostrou que esse apoio favoreceu a capacitação das equipes no acompanhamento medicamentoso, na avaliação clínica e discussão das necessidades do usuário, entre outras atividades ${ }^{(26)}$. Assim, as atividades de consultoria e ligação, realizadas pelos profissionais especializados em Saúde Mental e Psiquiatria, na perspectiva dos profissionais da ESF, podem apontar para as potencialidades de ampliação da capacidade técnica das equipes de saúde na ESF, corroborando estudos prévios ${ }^{(25-27)}$.

Em relação à elevada demanda de usuários que buscam a ESF para o tratamento em saúde mental, os achados deste estudo estão em concordância ao estudo multicêntrico, realizado em 14 países e coordenado pela Organização Mundial da Saúde(6), que mostrou que, em média, 24\% das pessoas atendidas em cuidados primários apresentavam, ao menos, um transtorno mental atual e outras $9 \%$ apresentavam condições subssindrômicas clinicamente significativas. De acordo com os depoimentos, os profissionais das equipes das ESF constataram que necessitam de educação permanente para aumentar o seu conhecimento teórico-técnico e as suas habilidades para o manejo de problemas de saúde mental devido à elevada demanda de pessoas com esses problemas que enfrentam no dia a dia.

Destaca-se que, dos 50 (100\%) participantes, 19 (38\%) eram agentes comunitários de saúde e constituem uma parcela significativa de profissionais de saúde mental que estão mais próximos dos usuários. Por outro lado, reconhece-se que os agentes comunitários ainda carecem de habilidades para lidar com as pessoas 
com transtorno mental. A falta de capacitação dos profissionais de saúde para lidar com os problemas de saúde mental pode produzir sofrimento psíquico e comprometer a resolutividade da intervenção(7).

De acordo com as diretrizes de atenção à saúde na ESF, o cuidado deve ser ampliado e deve acontecer no espaço social do indivíduo. Nessa vertente, a atenção integral e a reabilitação psicossocial são alguns dos pressupostos da atenção psicossocial que propiciam que as ações de saúde mental, realizadas na ESF, possam ser organizadas. Essa organização deve envolver os profissionais especializados na orientação, discussão e proposição de intervenções para a manutenção do cuidado às equipes da ESF(26). Assim, espera-se que a consultoria de Psiquiatria e ligação possa exercer um papel chave na integralidade do cuidado em saúde mental.

\section{Conclusão}

As atividades de consultoria e ligação mostraram-se como um espaço de aprendizado mútuo entre especialistas de Psiquiatria e Saúde Mental e equipes da ESF. Recomenda-se a ampliação do número de atividades que articulem as ações de saúde mental com a ESF, como a consultoria e ligação, a fim favorecer a sensibilidade das equipes para as demandas de saúde mental na ESF e consolidação da integralidade do cuidado. Além disso, tal atividade tem potencial para promover a interdisciplinaridade no trabalho cotidiano nos serviços de saúde mental e consolidar processos de trabalho mais potentes e resolutivos. No entanto, a consultoria e ligação são abordagens desafiadoras, pois constantemente questionam as fronteiras entre os níveis de atenção à saúde, conhecimentos, práticas, objetivos dos profissionais de saúde, entre outros.

\section{Referências}

1. Barban EG, Oliveira AA. O modelo de assistência da equipe matricial de saúde mental no Programa Saúde da Família do município de São José do Rio Preto. Arq Cienc Saúde. 2007;14(1):54-65.

2. Kate N, Craven M, Crustolo AM, Nikolaou L, Allen C. Integrating mental health services within primary care - a Canadian Program. Gen Hosp Psychiatry. 1997;19:324-32.

3. Gonçalves DM, Kapczinski F. Prevalência de transtornos mentais em indivíduos de uma unidade de referência para Programa Saúde da Família em Santa Cruz do Sul, Rio Grande do Sul, Brasil. Cad Saúde Pública. [Internet]. set 2008 [Acesso 12 fev 2018]; 24(9):2043-53. Disponível em: http://www.scielo.br/scielo.php?script $=$ sci_ arttext\&pid $=$ S0102-311X2008000900010\&Ing $=$ en. http://dx.doi.org/10.1590/S0102-311X2008000900010.
4. Goldberg D. Implications of epidemiological findings for the management of mental disorders encountered in primary care settings. Europ Psychiatry. 1997;12(suppl 2):56s-62s. https://doi.org/10.1016/ S0924-9338(97)80208-8

5. Einseberg L. Public Health Importance. In: Jenkins R, Ustun TB, editors. Preventing Mental Illness: Mental Health Promotion in Primary Care. Chichester (England): John Wiley \& Sons; 1998. p.141-53.

6. Organização Mundial da Saúde. Relatório sobre a saúde no mundo, 2001. Saúde Mental: nova concepção, nova esperança. Lisboa; 2002.

7. Gama CAP, Campos RO. Saúde mental na Atenção Básica- Uma pesquisa bibliográfica exploratória em periódicos de saúde coletiva (1997-2007). Cad Bras Saúde Mental. out/dez 2009;1(2):112-31.

8. Nunes M, Jucá VJ, Valentim CPB. Ações de saúde mental no Programa Saúde da Família: confluências e dissonâncias das práticas com os princípios das reformas psiquiátrica e sanitária. Cad Saúde Pública. out 2007;23(10):2375-84.

9. McWhinney IR. Philosophical and Scientific Foundations of Family Medicine (Chapter 5). In: Textbook of Family Medicine. New York (USA): Oxford University Press; 2003.

10. Borrel-Cárrio F, Suchman AL, Epstein RM. The Biopsychosocial Model 25 years later: principles, practice and scientific inquiry. Ann Fam Med. 2004; 2:576-82.

11. McDaniel SH, Hepworth J, DohertyWJ. Terapia Familiar Médica: um enfoque biopsicossocial às famílias com problemas de saúde mental. Porto Alegre; 1994.

12. Azevedo-Marques, J.M. Detecção e diagnóstico de transtornos mentais pela equipe de saúde da família. Tese [Doutorado]. Ribeirão Preto: Faculdade de Medicina de Ribeirão Preto da Universidade de São Paulo; 2009.

13. - Gillies D, Buykx P, Parker AG, Hetrick SE. Consultation liaison in primary care for people with mental disorders. Cochrane Database Syst Rev. 2015 Sep 18;(9):CD007193. doi: 10.1002/14651858. CD007193.pub2. Review. PubMed PMID: 26384252.

14. - Moscovici L, Azevedo Marques JM, Zuardi AW. 2016: Integrated mental healthcare: outcomes from Brazilian primary care. Eur J Person Centered Healthcare. 2016;4:669-74.

15. Moscovici L, Azevedo-Marques JM, Bolsoni LM, Rodrigues-Junior $A L$, Zuardi AW. Impact of different approaches of primary care mental health on the prevalence of mental disorders. Prim Health Care Res Dev. 2017 Dec 5;19(3):256-63.

16. Sharrock J, Grigg M, Happell B, Keeble-Devlin B, Jennings $S$. The mental health nurse: a valuable addition to the consultation-liaison team Int J Ment Health Nurs. 2006 Mar;15(1):35-43. 
17. Martin AC, Davis LL. Mental health problems in primary care: a study of nurse practitioners' practice. Nurse Pract. 1989;14(10):46-7,50-6.

18. Ministério da Saúde (BR). Secretaria de Atenção à Saúde, Coordenação de SM e Coordenação da Gestão da $A B$. $S M$ e $A B$ - O vinculo e o diálogo necessários Inclusão das ações de SM na AB. Circular conjunta n. 01/03 de 13/11/2003.

19. Munari DB, Melo TS, Pagotto V, Rocha BS, Soares CB, Medeiros M. Saúde Mental no contexto da atenção básica: potencialidades, limitações, desafios do Programa Saúde da Família. Rev Eletr Enferm. 2008;10(3):784-95. Disponível em: http://www.fen.ufg.br/revista/v10/n3/ v10n3a24.htm

20. Lucchese R, Oliveira AGB, Conciani ME et al. Mental health and the Family Health Program: pathways and obstacles in a necessary approach. Cad Saúde Pública. 2009;25(9):2033-42.

21. Ministério da Saúde (BR). Conselho Nacional de Saúde. Resolução no 196/96. Dispõe sobre normas de pesquisa envolvendo seres humanos. Brasília: MS; 1996. 24 p.

22. Bardin, L. Análise de Conteúdo. Lisboa (PT): Edições 70; 1977.

23. Campos GWS, Domitti AC. Apoio matricial e equipe de referência: uma metodologia para gestão do trabalho interdisciplinar em saúde. Cad Saúde Pública. 2007;23(2):399-407.

24. Cunha GT, Campos GWS. Apoio Matricial e Atenção Primária em Saúde. Saúde Soc. 2011;20(4):961-70.

25. Figueiredo MD, Campos RO. Saúde Mental na atenção básica à saúde de Campinas, SP: uma rede ou um emaranhado?. Ciênc Saúde Coletiva. fev 2009; [Internet]. [Acesso $12 \mathrm{fev} 2018]$; 14(1):129-38. Disponível em: http://www.scielo.br/scielo.php?script $=$ sci_ arttext\&pid $=$ S1413-81232009000100018\&Ing=en. http://dx.doi.org/10.1590/S1413-81232009000100018. 26. Mielke FB, Olchowsky A. Saúde mental na Estratégia Saúde da Família: avaliação de apoio matricial. Rev Bras Enferm. nov-dez. 2010;63(6):900-7.

27. Thielke S, Vannoy S, Unützer J. Integrating Mental Health and Primary Care. Primary Care: Clinics in Office Practice. 2007;34:571-92.
Recebido: 11.05.2017

Aceito: 07.12 .2018
Autor correspondente:

Ana Carolina Guidorizzi Zanetti

E-mail: carolzan@eerp.usp.br

(D) https://orcid.org/0000-0003-0011-4510
Copyright $\odot 2019$ SMAD, Rev. Eletrônica Saúde Mental Álcool Drog. Este é um artigo de acesso aberto distribuído sob os termos da Licença Creative Commons CC BY-NC.

Esta licença permite que outros remixem, adaptem e criem a partir do seu trabalho para fins não comerciais, e embora os novos trabalhos tenham de lhe atribuir o devido crédito e não possam ser usados para fins comerciais, os usuários não têm de licenciar esses trabalhos derivados sob os mesmos termos. 